\title{
LIDAR comparison for the use of orchard characterisation
}

\author{
T. Leseur, M. Hagedorn and R.L. Roten \\ Lincoln Agritech LTD, Engineering Dr, Lincoln 7640, New Zealand \\ Corresponding author: rory.roten@lincolnagritech.co.nz
}

LIDAR (Light Detection and Ranging) is a remote sensing technology that measures distance by illuminating a target with a laser and analysing the reflected light. LIDAR is largely used in geography, geology, forestry and other sectors. In agriculture LIDAR is used for creating topographic maps of fields and for crop mapping in orchards and vineyards. The objective of this study was to test two LIDAR systems for their ability to obtain useful canopy characteristics within an apple orchard. The two LIDAR systems included a SICK LMS200-30106 and a SICK LMS511-10100 PRO SR, which mainly differ by the laser beam divergence, the number of signal returns, the range, and the resolution as well as frequency values. Methods involved mounting each LIDAR side-by-side in conjunction with a RTK GPS and computer system. One orchard line was scanned on both sides in order to obtain a full scan at three differing ground levels: $60 \mathrm{~cm}, 115 \mathrm{~cm}$ and $120 \mathrm{~cm}$. As expected, the captured data indicate differing resolutions and further analysis is being administered to determine canopy characteristics between the two LIDAR models, such as percent open space and porosity. This technology could be used for improving agrichemical application in orchards.

\section{Evaluation of spray drift with an experimental ultrasonic sensor sprayer in a dwarf apple orchard}

\author{
R.L. Roten ${ }^{1}$ and J.P. Praat ${ }^{2}$ \\ ${ }^{1}$ Lincoln Agritech, Engineering Dr, Lincoln 7640, New Zealand \\ ${ }^{2}$ Groundtruth Ltd, 104 Turere Lane, Te Awamutu, New Zealand \\ Corresponding author: rory.roten@lincolnagritech.co.nz
}

Dwarf apple trees are becoming more common in New Zealand due to their easier maintenance and more efficient production. However, this may increase the risk of spray drift if orchardists do not adjust spraying practices to match shorter dwarf varieties of fruit trees as compared with larger more traditional canopies as dwarf trees have less foliage to intercept spray. A study was carried out to examine the off-target movement of the spray plume from a conventional air-blast sprayer as compared with an experimental ultrasonic sensor sprayer in a dwarf apple canopy. The experimental sensor sprayer was set up to respond to the target canopy. Three treatments were carried out including a conventional Typhoon 1500 orchard sprayer and a single row ultrasonic sensor sprayer with either sensors ON or OFF. Each sprayer was set to deliver 500 litres/ha of a $0.4 \mathrm{~g} /$ litre concentration of a fluorescent dye (PTSA, 1,3,6,8-pyrenetetrasulfonic acid tetrasodium salt). The drift was quantified by using a series of mylar cards, Petri dishes and fishing nylon. Results indicated a $24 \%$ decrease of deposited drift from 1 to $100 \mathrm{~m}$ with the sensors turned on and a 5\% increase with the sensors off as compared to the conventional sprayer. 